\title{
Comparison of Different Diagnostic Method for Mycobacteria Tuberculosis in Suspected Patients
}

\author{
Khagi AR, ${ }^{1}$ Singh S, ${ }^{1}$ Subba S, ${ }^{1}$ Bajracharya A, ${ }^{1}$ Tuladhar $R,{ }^{1}$ Lekhak B, ${ }^{1}$ Shrestha KB ${ }^{2}$ \\ ${ }^{1}$ Central Department of Microbiology, Tribhuvan University, Kirtipur, Kathmandu, ${ }^{2}$ National Tuberculosis Center , Thimi, \\ Bhaktapur, Nepal
}

\section{ABSTRACT}

Background: Microbial examination of smear of AFB by Z-N stain is currently the most rapid method for the detection of $M$. tuberculosis but its sensitivity is low i.e. required at least 10,000 bacterial cells per ml of sputum and also none specific, but auramine staining method has higher sensitivity than that of the Z-N stain but there are chances of false positive. Objective of this study was to find the correlation between chest X-ray, direct sputum smear examination by Ziehl-Neelsen stain, Auramine fluorochrome stain and sputum culture for M. tuberculosis.

Methods: During that study period 250 x 3 samples were taken three each from 250 patients and divided into two groups A and B by performing Auramine fluorochrome stain in all samples . In group A, there were 150 fluorochrome stain positive samples. One each from 150 patient for comparative study of direct sputum smear examination by Ziehl-Neelsen stain, , culture on LJ medium and chest X-ray. Similarly in group B, next 100 fluorochrome stain negative specimens one each from 100 patients were taken for the comparative study of direct sputum smear examination by Ziehl-Neelsen stain, culture and chest X-ray.

Results: In the study group A $(n=150)$ all the specimens were positive in Auramine fluorochrome stain and all of them show positive in X-ray but only 134 showed positive in Ziehl-Neelsen stain and 136 showed positive in culture. In the study group $B(n=100)$, all the specimens were negative in Auramine fluorochrome stain and all of them show negative in Ziehl-Neelsen stain but 14 of them were positive in culture and 24 were positive in chest X-ray.

Conclusions: The diagnosis of PTB could be made by Auramine fluorochrome microscopy and culture.

Key words: auramine fluorochrome stain, culture, mycobacterium tuberculosis, x-ray, ziehl-neelsen.

\section{INTRODUCTION}

In Nepal, $45 \%$ of the total population is infected with $\mathrm{TB}$, out of which $60 \%$ are in the productive age group (15-45). Every year 40,000 people develop active TB, of whom 20,000 have infectious pulmonary disease. ${ }^{1}$

Due to the risk of the spread of disease and the potential for the emergence of drug resistant strains, the rapid diagnosis of the TB is very important. Concerning detection of TB, the lab diagnosis of TB relies on direct smear examination of sputum by Ziehl-Neilsen or Auramine fluorochrome stain and culture. It is also aided by the chest $X$-ray. Microbial examination of smear of AFB by Z-N stain is currently the most rapid method for the detection of $M$. tuberculosis but its sensitivity is low i.e. required at least 10,000 bacterial cells per $\mathrm{ml}$ of sputum and also none specific, but auramine staining method has higher sensitivity than that of the Z-N stain but there are chances of false positive. Isolation of the organism by culture and identification by bio-chemical test is much more sensitive and specific but is time consuming. ${ }^{2}$

Correspondence: Mr. Sudeep Singh, Central Department of Microbiology, Tribhuvan University, Kirtipur Kathmandu. Phone: 9803020651, Email: sudeeporma@gmail.com. 
Comparative Study of Different Diagnostic Method for Mycobacteria Tuberculosis in Suspected Patients

\section{METHODS}

A Cross-sectional study was conducted at National Tuberculosis Centre, Thimi, Bhaktapur, Nepal from June 29, 2005 to December 29, 2005. A total of 250 samples were included in the study. Ethical approval and consent of patient was taken. Sputum is the sample of choice in this study. The samples were divided into group $A$ by Sputum smear positive by Auramine fluorochrome stain $(n=150)$, one from each patient and group B by Sputum smear negative by Auramine fluorochrome stain $(n=100)$, one from each patient.

The sputum sample was the subjected to direct microscopy examination by Zeil-Neilson and Auramine fluorochrome method. In ZN staining the Acid fast bacteria (AFB) appear red and they were reported and recorded according to the Bulletin of the International Union Against Tuberculosis (IUATLD) 1978 WHO. Similarly in auramine method the reporting and recording was done according to the ALA scale (American Lung Association, USA).

Another part of the sample was used for primary culture by Lowstein-Jension method. The inoculated slants were placed in the incubator at $37^{\circ} \mathrm{C}$. The caps were closed tightly when the surface of the media dried; incubation was continued up to at least 8 weeks. The culture was observed at the 7 day for the rapid growers and at fourth week for slow growers.

Presence of colonies on the medium was checked when colonies were present at each stage (at $7^{\text {th }}$ day or $4^{\text {th }}$ week); their acid fastness was determined by Z$\mathrm{N}$ staining. If the colonies do not appear at the time mentioned above, observe weekly until 8 weeks before giving decision as negative. Recording and reporting by was done according to WHO guidelines.

Chest X-ray was taken by radiographer technologist of NTC and reading of X-ray was done by expert of NTC. In National Tuberculosis Centre, mainly mass miniature $X$ ray film was taken for tuberculosis of lung, which could be used to diagnose PTB with certain characteristics of chest radiograph. Following criteria of radiography appearance are used for diagnosis of tuberculosis in NTC, shadow in upper zone, nodular shadow, and presence of cavity. Bilateral shadows and the persistence of abnormal shadows without alternation in an X-ray should be further investigated with other diagnostic tools such as Bronchoscopy, CT scan etc.

\section{RESULTS}

In the study group A $(n=150)$ all the specimens were positive in Auramine fluorochrome stain and all of them show positive in X-ray but only 134 showed positive in
Ziehl-Neelsen stain and 136 showed positive in culture( Table 10).

In the study group $B(n=100)$, all the specimens were negative in Auramine fluorochrome stain and all of them show negative in Ziehl-Neelsen stain but 14 of them were positive in culture and 24 were positive in chest $X$-ray. The study was not verified by PCR.

The group A includes 150 direct Auramine fluorochrome smear positive sputum samples, $90.66 \%$ of them were positive in cultural examination in LJ medium, $89.33 \%$ were positive in Ziehl-Neelsen stain and all the specimens i.e. $100 \%$ were positive in radiological examination.

Among the studied 150 Auramine fluorochrome stain positive cases, $82 \%(n=123)$ were male and $18 \%(n=27)$ were female. This study showed that the highest number was seen in the age group 41 to 50 (26.66\%), followed by 31 to 40 (24\%) (Table 2).

Out of 150 Auramine fluorochrome positive cases 123 (82\%) were male and 27 (18\%) were female. This greater occurrence in male than female is statistically significant $(\mathrm{x} 2=61.44)$.

Among the studied 150 Auramine fluorochrome stain positive cases, only 136 cases were positive in culture, in which $82 \%(n=111)$ were male and $18 \%(n=25)$ were female. This study showed that the highest number was seen in the age group 31 to $40(26.47 \%)$, and 41 to 50 (26.47\%), followed by 21 to 30 (18.38\%) (Table 3). Among the studied 150 Auramine fluorochrome stain positive cases, all 150 cases were positive in X-Ray, in which 82\% $(n=123)$ were male and $18 \%(n=27)$ were female. This study showed that the highest number was seen in the age group 41 to 50 (26.66\%) (Table 4$)$.

Out of 150 Auramine Fluorochrome positive cases 123 (80\%) were male and 27 (18\%) were female. This greater occurrence in male than female is statistically significant $(x 2=61.44)$.

Among the studied 150 Auramine fluorochrome stain positive cases, all 134 cases were positive in ZiehlNeelsen stain, in which $82 \%(n=123)$ were male and $18 \%$ $(\mathrm{n}=27)$ were female. This study showed that the highest number was seen in the age group 41 to $50(27.61 \%)$, followed by 31 to 40 (26.86\%), 21 to 30 (15.67\%), 11 to $20(14.92 \%)$ and so on.

Out of 134 Ziehl-Neelsen stain positive 110 (82\%) were male and 24 (18\%) were female. This greater occurrence in male than female is statistically significant $(x 2=$ 55.18).

In group B, out of 100, Auramine fluorochrome smears negative sputum samples, $24 \%$ (24/100) were found to 
be positive by X-ray and the remaining $76 \%(76 / 100)$ were negative, where all fluorochrome stain positive samples showed positive in Z-N stain (Table 5 ).

Among $100(100 \%)$ Auramine fluorochrome staining negative sputum samples collected from these cases, $14 \%(14 / 100)$ samples were positive by culture in LJ medium where as the remaining $86 \%$ were negative.

\section{DISCUSSION}

According to the WHO guidelines for TB control, patient with more than three weeks history of cough should be screened for pulmonary tuberculosis with direct sputum smear examination for M. tuberculosis. Because the clinical signs and symptoms of pulmonary tuberculosis are not specific, the fundamental principal for the diagnosis of tuberculosis is the accurate demonstration of $M$. tuberculosis in a suitable specimen from the suspected cases of PTB for the adequate treatment. Either presumptive diagnosis is based on the demonstration of tubercle bacilli in the sputum or. tuberculosis may be demonstrated by culture. In case of pulmonary tuberculosis, X-ray examinations have been used frequently as a primary screening method. ${ }^{3}$

The added advantages of sputum smear microscopy is that it has very close relation with infectiousness, patient who are sputum smear positive and culture positive are for more likely to be infectious than culture positive but smear negative. ${ }^{4}$

In this comparative study, the total specimen studied were 250x3, three from each clinically suspected pulmonary tuberculosis patients. These specimens were examined by direct sputum microscopy by Auramine fluorochrome stain, Ziehl-Neelsen stain and Culture. These specimens were divided in two groups $A$ and $B$. In group $A(n=150)$ one specimen from each patient were taken which all were positive in Auramine fluorochrome stain and compared with chest X-ray, culture and ZiehlNeelsen stain. Similarly in group $B(n=100)$ one specimen from each patient were taken which all were negative in Auramine fluorochrome stain and compare with chest $\mathrm{x}$-ray, culture and Ziehl-Neelsen stain.

In group A all the specimens were positive in direct sputum microscopy examined by Auramine fluorochrome stain, out of 150 samples only 136 were positive in culture and 134 were positive in direct sputum smear microscopy examined by Z-N stain but all the Auramine fluorochrome stain positive cases showed positive in chest x-ray report, which were read by expert of NTC (National Tuberculosis Center) Thimi, Bhaktapur, Nepal.

In the study group A, 14 patient suspected of PTB were negative in culture for MTB so there may be certain other causes which are responsible for common symptoms to tuberculosis. The reason for negative result in culture may be due to various reasons. ${ }^{5}$

The efficacy of chest radiography is determined largely by the reader's ability to detect abnormal opacities and to interpret them correctly. ${ }^{5}$ This implies not missing or under reading they are, conversely, not over reading normal opacities on the film. This ability varies from one reader to another.

Similar results were obtained by Shrestha German Nepal TB project, Katmandu. Among 1280 PTB suspected cases only 67 cases were sputum smear positive as examined by fluorescent microscopy whereas only $14 \%$ of the positive cases were culture positive. Similarly in a study conduced among 100 suspected cases of PTB by chest $\mathrm{X}$-ray, only 82 were sputum smear positive examined by fluorochrome microscopy and $59.7 \%$ of the sputum smear positive cases were culture positive. ${ }^{7}$

In our study, 150 subjects all having the positive in sputum smear examined by fluorochrome stain, only 136 of the sputum smear positive cases were culture positive.

Another study was conducted by Nagpaul in the National tuberculosis institute, Bangalore, India. In this study 2229 outpatients were randomly selected with chest symptoms. 227 were classified by $\mathrm{X}$-ray as tuberculosis but 81 of them were not confirmed by bacteriological examination. Among the remaining 2002 patients classified as normal or as having a disease other than tuberculosis, 31 of them were found culture positive and smear microscopy. On the other hand among 145 patients positive by smear 130 were confirmed by culture.

In this study out of 150 microscopy smear positive by Auramine fluorochrome stain $90.66 \%$ were culture positive and $M$. tuberculosis is the main causative agent so we could say that $M$. tuberculosis is the main causative agent of tuberculosis among the Nepalese population. MOTT and $M$. bovis were not isolated.

Similarly in study group A among 150 direct sputum smear positive by Auramine fluorochrome stain only 134 were positive in direct sputum smear positive by Z-N method and 16 cases were negative.

Similarly in our study group A among 150 cases of direct sputum smear positive in Auramine fluorochrome stain all cases were positive in chest $\mathrm{X}$-ray examined by expert of the NTC, Thimi, Bhaktapur.

The criteria of chest radiograph favoring the diagnosis of tuberculosis in NTC. ${ }^{8}$

Shadow mainly in the upper zone, Patch or nodular shadow. The presence of a cavity or cavities, although 
carcinoma or pneumonia may occur in an area of the lung where there is calcification due to TB. Bilateral shadows, especially if there are in upper zone. The persistence of abnormal shadows without alternation in an X-ray should be further investigated.

\begin{tabular}{|c|c|c|}
\hline Method & Positive cases & Negative cases \\
\hline Fluorescent method & 150 & 00 \\
\hline X-ray & 150 & 00 \\
\hline Culture & 136 & 14 \\
\hline Ziehl-Neelsen method & 134 & 16 \\
\hline
\end{tabular}

Table 2. Distribution of Auramine fluorochrome stain positive cases by age and sex

\begin{tabular}{|c|c|c|c|}
\hline $\begin{array}{l}\text { Age group } \\
\text { (year) }\end{array}$ & Male & Female & Total \\
\hline- & No. & No. & No. \\
\hline $0-10$ & - & - & - \\
\hline $11-20$ & 21 (14) & $3(2)$ & $24(16)$ \\
\hline $21-30$ & 25 (16.6) & $4(2.66)$ & $29(19.33)$ \\
\hline $31-40$ & $28(18.6)$ & $8(5.33)$ & $36(24)$ \\
\hline $41-50$ & $34(22.66)$ & $6(4.00)$ & $40(26.66)$ \\
\hline $51-60$ & $4(2.66)$ & $3(2)$ & $7(4.66)$ \\
\hline $61-70$ & $6(4)$ & $2(1.33)$ & $8(5.33)$ \\
\hline $71-80$ & $2(1.33)$ & $1(0.66)$ & $3(2)$ \\
\hline $81-90$ & $3(2)$ & 0 & $3(2)$ \\
\hline Total & $123(82)$ & 27 (18) & $150(100)$ \\
\hline
\end{tabular}

\begin{tabular}{|c|c|c|c|}
\hline Age group (year) & Male & Female & Total \\
\hline - & No. & No. & No. \\
\hline $0-10$ & - & - & - \\
\hline $11-20$ & 19 (13.97) & $2(1.47)$ & $21(15.57)$ \\
\hline $21-30$ & $22(16.18)$ & $3(2.2)$ & $25(18.38)$ \\
\hline $31-40$ & $28(20.58)$ & $8(5.88)$ & $36(26.47)$ \\
\hline $41-50$ & $30(22.06)$ & $6(4.41)$ & $36(26.47)$ \\
\hline $51-60$ & $4(2.94)$ & $3(2.2)$ & $7(5.15)$ \\
\hline $61-70$ & $6(4.41)$ & $2(1.47)$ & $8(5.88)$ \\
\hline $71-80$ & $2(1.47)$ & $1(0.73)$ & $3(2.20)$ \\
\hline $81-90$ & - & - & - \\
\hline Total & $111(82)$ & $25(18)$ & 136 \\
\hline
\end{tabular}

\begin{tabular}{|c|c|c|c|}
\hline Age group (year) & $\begin{array}{l}\text { Male } \\
\text { No. }\end{array}$ & $\begin{array}{c}\text { Female } \\
\text { No. }\end{array}$ & $\begin{array}{l}\text { Total } \\
\text { No. }\end{array}$ \\
\hline $0-10$ & - & - & - \\
\hline $11-20$ & $18(13.43)$ & $2(1.49)$ & $20(14.92)$ \\
\hline $21-30$ & $19(14.18)$ & $2(1.49)$ & $21(15.67)$ \\
\hline $31-40$ & $28(20.89)$ & $8(5.97)$ & $36(26.86)$ \\
\hline $41-50$ & $31(23.13)$ & $6(4.47)$ & $37(27.61)$ \\
\hline $51-60$ & $4(2.98)$ & $3(2.23)$ & $7(5.22)$ \\
\hline $61-70$ & $5(3.73)$ & $2(1.49)$ & $7(5.22)$ \\
\hline $71-80$ & $2(1.49)$ & $1(0.75)$ & $3(2.24)$ \\
\hline $81-90$ & $3(2.23)$ & 0 & $3(2.24)$ \\
\hline Total & $110(82.0)$ & $24(18)$ & 134 \\
\hline
\end{tabular}

\begin{tabular}{|c|c|c|c|}
\hline Methods & Total cases & positive & negative \\
\hline $\begin{array}{l}\text { Fluorescent } \\
\text { microscopy }\end{array}$ & 100 & 000 & 100 \\
\hline $\begin{array}{l}\text { Ziehl-Neelsen } \\
\text { microscopy }\end{array}$ & 100 & 000 & 100 \\
\hline X-Ray & 100 & 24 & 76 \\
\hline Culture & 100 & 14 & 86 \\
\hline
\end{tabular}

Pulmonary TB may mimic many other diseases and difficulty in diagnosis may arise under the following circumstances.

Bronchogenic carcinoma may be suggested in middle aged smoker by evidence of collapse in the upper lobes or the presence of a solitary round focus. Tomographs showing central cavitations, calcification or the presence of satellite lesions. Although favoring tuberculosis, may also occur in carcinoma. Although favoring tuberculosis may also occur in carcinoma. In the absence of positive sputum conformation of diagnosis may require histological evidence from bronchial biopsy, transbronchial or needle biopsy of the lung or occasionally throractomy. Even the finding of tubercle bacilli does not exclude carcinoma. Since both diseases may coexist and falsely positive sputum cytology may occur in the presence of active pulmonary tuberculosis.

In acute pneumonia and lung abscess, examination of sputum for acid fast bacilli should not be forgotten, especially if these conditions do not respond to antibiotics chronic pneumonia due to staphylococci, 
Klebsiella pneumonia or anaerobic organisms can result in an illness resembling tuberculosis. The presence of much foul sputum suggests pyogenic abscess.

Sarcoidosis-patients suffering from sarcoidosis typically have few symptoms of tuberculosis and negative in tuberculin tests, confirmations of such patients is done by biopsy of lymph node, kvein test site or lung will establish the diagnosis. It should be remembered that tissue taken from the periphery of caseating tuberculous lesions may show non-caseating granulomata histologically indistinguishable from sarcoidosis.

Allergic bronchopulmonary aspergillosis of lung standing gives rise to fix upper zone shadows resulting from bronchiaectasis and fibrosis. This appearance closely resembling tuberculosis. Patients are usually asthmatic and have characteristic immunological evidence of allergic aspergillosis.

Extrinsic alveolitis, especially avian gives upper lobe shadowing but should be distinguished by a history of exposure to organic antigens and by immunological tests.

Pneumococosis, especially that due to silica or cola may be complicated by tuberculosis and require investigation by careful sputum examination.

The chronic constitutional symptoms associated with pulmonary tuberculosis may be mistaken for those produced by many other disorders, including psychoneurosis, diabetes mellitus and hyperthyroidism and in the elderly may simply be attributed to old age; tuberculosis should never be forgotten as an important cause of fever of obscure origin.

\section{CONCLUSIONS}

Since all the Auramine fluorochrome stain positive cases showed positive in X-ray and all the Auramine fluorochrome stain negative cases showed negative in Z$\mathrm{N}$ stain, the Auramine fluorochrome stain positive cases do not require to do $X$-ray examination and Auramine negative cases do not require to do $\mathrm{Z}-\mathrm{N}$ stain, it saves time and money but some of the Auramine negative cases showed positive in culture. Hence the study concluded that the diagnosis of PTB could make by Auramine fluorochrome microscopy and culture. Not all $X$-ray shadows, collapse, cavities etc should be treated for PTB unless Sputum is positive. Depending upon other supporting symptoms further investigations are recommended.

\section{ACKNOWLEDGEMENTS}

I am especially thankful to Mr. Dhruba Kumar Khadka, Mr. Jagat Khadka, Mr. Bhola Chaudhary, Mr. Ram Babu Shrestha and all other staffs of National Tuberculosis Centre and SAARC Tuberculosis Centre, for their kind help and support and to my friends Rama Amatya, Luma Shrestha and Bima Shrestha. I am equally thankful to Mr. Aryan Babu Shrestha, Mr. Bikash Shakya.

\section{REFERENCES}

1. National Tuberculosis Centre (NTC). Annual Report. National tuberculosis programme; 2002/2003.

2. Chakraborty P. Mycobacterium tuberculosis. In: A text book of microbiology 2nd ed. India: New Central Book Agency (P) Ltd; 2003;396-414.

3. Suitters BT, Brogger SA. Some aspects of laboratory investigations in a mass campaign against tuberculosis. Bull. World Hlth Orgn. 1967;36:837-45.

4. Narain R, Rao MS. Chandrashekhar P. Microscopy positive and microscopy negative cases of pulmonary tuberculosis. Am Rev Respire Dis. 1971;103:761-3.

5. Jain A, Bhargava A, Agrawal SK. A comparative study of two commonly used staining techniques for acid fast bacilli in clinical specimens. Int J Tub. 2002;49:161-2.

6. Toman K. Tuberculosis case finding and chemotherapy. New Delhi, India: Jaypee Brothers; 1989

7. Bhatta CP. Study of different diagnostic methods and prevalence of pulmonary tuberculosis among Nepalese population. 1996.

8. Prasai MK. X-ray as a method of screening at National tuberculosis center. Seminar and workshop on national tuberculosis control program, Thimi, Bhaktapur, Nepal, 1992. 